\title{
Infections and vaccinations as risk factors for childhood Type I (insulin-dependent) diabetes mellitus: a multicentre case-control investigation
}

\author{
The EURODIAB Substudy 2 Study Group*
}

\section{Abstract}

Aims/hypothesis. To determine if vaccinations and infections are associated with the subsequent risk of Type I (insulin-dependent) diabetes mellitus in childhood.

Method. Seven centres in Europe with access to population-based registers of children with Type I diabetes diagnosed under 15 years of age participated in a case-control study of environmental risk factors. Control children were chosen at random in each centre either from population registers or from schools and policlinics. Data on maternal and neonatal infections, common childhood infections and vaccinations were obtained for 900 cases and 2302 control children from hospital and clinic records and from parental responses to a questionnaire or interview.

Results. Infections early in the child's life noted in the hospital record were found to be associated with an increased risk of diabetes, although the odds ratio of 1.61 ( $95 \%$ confidence limits $1.11,2.33$ ) was significant only after adjustment for confounding variables. None of the common childhood infectious diseases was found to be associated with diabetes and neither was there evidence that any common childhood vaccination modified the risk of diabetes. Pre-school day-care attendance, a proxy measure for total infectious disease exposure in early childhood, was found, however, to be inversely associated with diabetes, with a pooled odds ratio of 0.59 (95\% confidence limits $0.46,0.76)$ after adjustment for confounding variables.

Conclusion/interpretation. It seems likely that the explanation for these contrasting findings of an increased risk associated with perinatal infections coupled with a protective effect of pre-school day care lies in the age-dependent modifying influence of infections on the developing immune system. [Diabetologia (2000) 43: 47-53]

Keywords Type I (insulin-dependent) diabetes mellitus, epidemiology, risk factors, case-control study, perinatal, infection, vaccination, child care.
From as long ago as the introduction of insulin therapy a possible role of infections in the onset of childhood diabetes has been suspected [1]. Infections in the months $[2,3]$ or year $[4,5]$ preceding diagnosis are more common in children with Type I (insulin-de-

Received: 12 July 1999 and in revised form: 27 August 1999

Corresponding author: Dr. C.C. Patterson, Department of Epidemiology and Public Health, The Queen's University of Belfast, Royal Victoria Hospital, Grosvenor Road, Belfast BT12 6BJ, UK

* see Acknowledgements pendent) diabetes mellitus than in control children and seasonal variation in onset with a peak in the winter months is evident throughout Europe [6]. This suggests that infections could play a part in precipitating Type I diabetes. The finding that a high proportion of children with fetal rubella embryopathy syndrome develop diabetes [7] suggests that infections early in life can also initiate autoimmunity. More recent observations indicate that coxsackie virus B infection during pregnancy is associated with an increase in diabetes risk [8-10].

Infectious diseases could be involved in the pathogenesis of autoimmune disease like diabetes in different ways. One is to directly attack the beta cell, an- 
other by affecting the developing immune system thus interfering with the future self/non-self discrimination capacity. This latter idea has been elaborated by immunologists based on experimental studies suggesting that infrequent infections and more widespread use of vaccinations lead to an increased risk of both atopic disease and childhood diabetes [11]. Other work from animal models has suggested that vaccinations can reduce the risk of diabetes [12]. Vaccinations have therefore been investigated as possible modulators of the risk of childhood diabetes. Several authors have looked for trends in childhood diabetes incidence rates after changes in countrywide vaccination policies. No detectable effect on incidence of Type I diabetes was reported after removal of either bacille Calmette-Guerin (BCG) [13] or pertussis [14] from the Swedish national immunisation programme. Although the elimination of mumps by a vaccination programme in Finland has been linked to the subsequent arrest of the incidence increase in diabetes among children aged 5-9 years, the incidence among children aged 0-4 years has continued to rise [15]. A recent analysis of diabetes incidence up to the age of 10 years in relation to differing Haemophilus influen$z a e$ type $\mathrm{b}$ vaccination regimes in Finland suggested that this vaccination and its timing were unlikely to be relevant [16]. The findings from various case control studies of vaccinations have been inconsistent. One study found mumps vaccination to be associated with a non-statistically significant reduction in diabetes risk [2], whereas another reported that measles vaccination, either alone or as a combined measles/ mumps/rubella vaccination, was associated with a risk reduction [4]. Vaccination of BCG has been reported not to be associated with subsequent diabetes risk, although the data were suggestive of a possible protective effect under the age of 5 years [17]. Two other studies have reported that no vaccination was statistically significantly associated with risk [3, 18]. The findings of these case-control studies are difficult to interpret because vaccination uptake rates in some countries are so high that comparisons between cases and controls lack power. Also, isolated statistically significant findings are to be expected as a consequence of the multiple comparisons inherent in such studies, and should only be given credence if they can be replicated.

As part of a large, multicentre, population-based, case-control study to investigate early environmental exposures as possible risk factors for Type I diabetes, we have collected data on both vaccinations and infectious diseases in children who were diagnosed with diabetes before the age of 15 years and in an age-matched group of control children. We also report findings on perinatal infections, on some specific childhood infections and on a proxy measure of exposure to infections based on attendance at pre-school groups.

\section{Methods and study design}

Each of the eight participating centres had a population-based register of childhood onset diabetes operating in accordance with the standards of the EURODIAB ACE Group [19]. Cases were therefore obtained from a temporally and geographically well-defined study base in each centre. After consultation with the study coordinators, a population-based sample of control children, matched to the cases in age distribution, was obtained in each centre using sources which depended on local circumstances as previously described [20]. One centre (Bulgaria) had difficulty in complying with this element of the study and is excluded from this report. In the remaining seven centres 1028 children with diabetes onset before the age of 15 years and 3044 control children were invited to participate. Of these, 900 children with diabetes and 2302 control children participated giving response rates of $87.5 \%$ and $75.6 \%$, respectively. A breakdown of numbers and response rates by centre is shown in Table 1 .

An agreed set of core variables was then collected either by interview (Latvia, Luxemburg, Romania, UK-Leeds) or by questionnaire (Austria, Lithuania and UK-Northern Ireland). Information about maternal/perinatal infections and antibiotic treatment was obtained both from hospital records and from parental recall. Vaccination information was considered to be validated if obtained by the investigator from an official source or from a contemporary entry in a child health care booklet kept by the parent. If the parent was, however, able to provide exact dates of vaccination this was accepted as evidence of the existence of a contemporary record and the vaccination was considered to be validated even if the record was not actually seen by the investigator. Vaccination data obtained solely from parental recall was considered to be unvalidated. The child's history of five specific infectious diseases was obtained from parental recall. Pre-school group attendance was defined as regular attendance at a day-care centre, nursery or playgroup on three or more days a week for a minimum of 1 year. All information was transferred to a standardised coding sheet and anonymous records were dispatched to a single centre for data preparation and analysis.

Local study leaders received detailed written instructions for the selection of control children, the conduct of interviews and the completion of the record sheet. Each centre was sitevisited by a study coordinator and centre leaders participated in workshops to maintain uniformity in study standards. All centres adhered to the principle of obtaining informed consent, and the approval of local research ethics committees was obtained where they existed.

All data on exposure to vaccinations and infectious diseases were corrected back to date of diagnosis for the children with diabetes or a corresponding date for control children obtained as the midpoint of the centre's period of recruitment of children with diabetes. The analysis of pre-school care was restricted to children with disease diagnosed after their fifth birthday since the day care arrangements made for children diagnosed before that age might have been influenced by their disease. Data from only six centres contributed to the analysis of pre-school care because one of the centres had already reported a more detailed analysis of its data [21].

The Mantel Haenszel approach was used to pool the results across centres, separate odds ratios being obtained for each centre, and these being combined using a weighting based on the numbers of children with diabetes and control children in the centre and their rates of exposure [22]. In addition to a test of significance on the combined odds ratio, a test for heterogeneity was also obtained which provides a comparison of the separate odds ratio between the centres. To adjust for potential 
Table 1. Summary of participants in the seven study centres

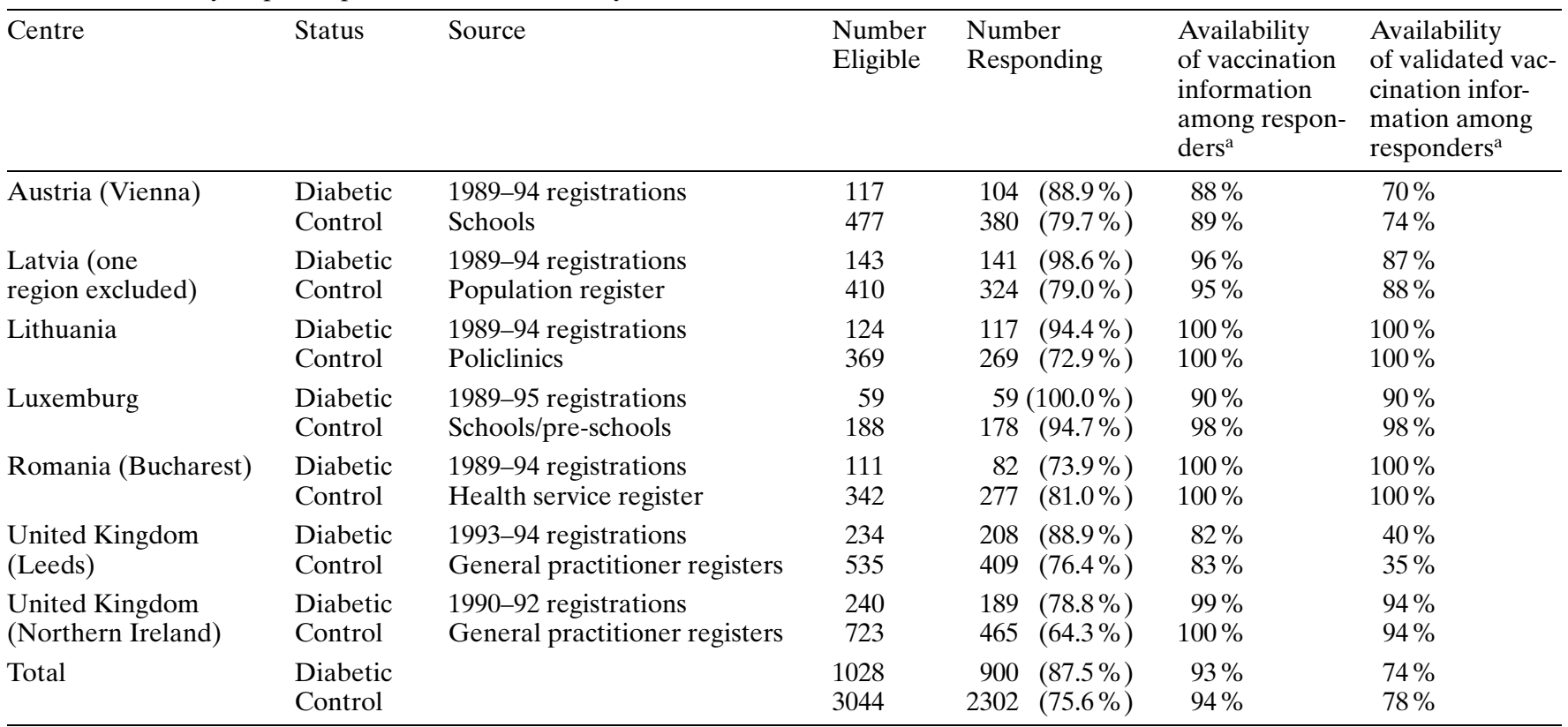

${ }^{a}$ Median of nine common vaccinations

confounders, logistic regression analysis was used with terms included in the model to represent centres. Statistical analyses were done using the SPSS and STATA (Stata Statistical Software, Release 6.0, Stata Corporation, College Station, Tex., USA) packages. A $p$ value of less than 0.05 was considered significant in all tests.

\section{Results}

The Mantel Haenszel pooled odds ratios for nine vaccinations are presented in the first column of Table 2. None of the odds ratios was statistically significant and there was no evidence of heterogeneity between centres. The corresponding odds ratios obtained from logistic regression analysis adjusting for confounding variables are shown in the second column, and again none attained statistical significance.
The analyses of data on maternal and perinatal infections is summarised in Table 3. Maternal infections, whether recalled by the mother or recorded in the hospital notes, were not associated with any significant elevation in the child's risk of diabetes. In contrast, infections in the newborn child, particularly if recorded in the hospital notes, were associated with an increased risk although the finding only attained significance after adjustment for variables with the potential to confound the association [20, 23]. Birth weight was the most influential confounder, with low birthweight infants more frequently being recorded as having infections. The full extent of the excess risk of diabetes associated with infections in the newborn therefore only became apparent when the reduced risk of diabetes in low birth weight infants observed in our study was taken into account.

Table 2. Odds ratios for nine common vaccinations before and after adjustment for confounding variables

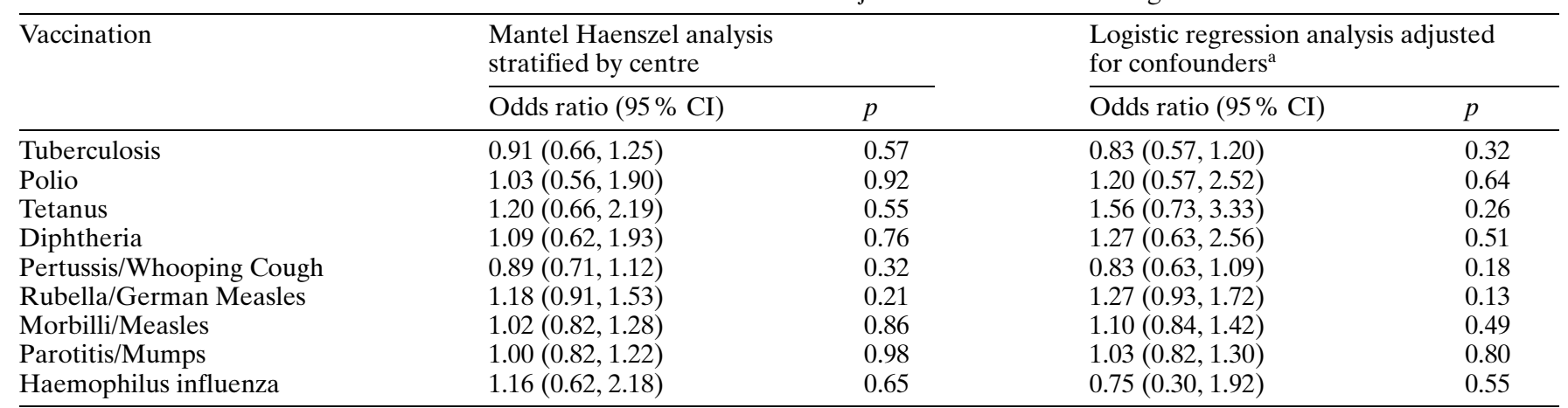

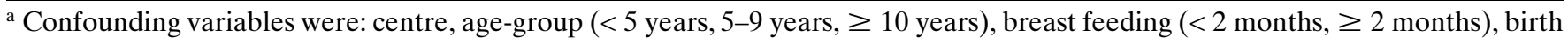
weight $(<2500 \mathrm{~g}, \geq 2500 \mathrm{~g})$, maternal age ( $\leq 25$ years, $>25$ years), jaundice at birth (yes, no), asthma before disease diagnosis (yes, no) and vitamin D supplementation (yes, no, unknown) 
Table 3. Odds ratios for maternal and neonatal infections and antibiotic treatment, before and after adjustment for confounding variables

\begin{tabular}{|c|c|c|c|c|}
\hline & \multicolumn{2}{|c|}{$\begin{array}{l}\text { Mantel Haenszel analysis } \\
\text { stratified by centre }\end{array}$} & \multicolumn{2}{|c|}{$\begin{array}{l}\text { Logistic regression analysis adjusted } \\
\text { for confounders }{ }^{\mathrm{a}}\end{array}$} \\
\hline & Odds ratio $(95 \% \mathrm{CI})$ & $p$ & Odds ratio $(95 \% \mathrm{CI})$ & $p$ \\
\hline $\begin{array}{l}\text { Maternal infection other than urinary tract } \\
\text { infection during pregnancy (hospital notes) }\end{array}$ & $1.07(0.82,1.40)$ & 0.61 & $1.03(0.78,1.37)$ & 0.82 \\
\hline $\begin{array}{l}\text { Antibiotic treatment during pregnancy } \\
\text { (hospital notes) }\end{array}$ & $1.22(0.87,1.73)$ & 0.25 & $1.25(0.87,1.79)$ & 0.24 \\
\hline $\begin{array}{l}\text { Severe neonatal infection in child } \\
\text { (maternal recall) }\end{array}$ & $1.39(0.91,2.13)$ & 0.13 & $1.58(0.97,2.57)$ & 0.07 \\
\hline $\begin{array}{l}\text { Neonatal infection in child } \\
\text { (hospital record) }\end{array}$ & $1.29(0.92,1.81)$ & 0.14 & $1.61(1.11,2.33)$ & 0.01 \\
\hline
\end{tabular}

The analysis of common childhood infections is summarised in Table 4. For none of the five specific infections considered was there evidence that the odds ratios was statistically significant. For morbilli this non-significant pooled odds ratio concealed, however, some heterogeneity between centres, significantly elevated odds ratios of 2.02 (95\% CI 1.18 , 3.47) for the Austrian centre and 3.50 (1.85, 6.03) for the Romanian centre being balanced by a nonsignificantly reduced odds ratio of $0.70(0.47,1.02)$ in the UK (Northern Ireland) centre. No obvious explanation can be found to explain this result. The pooled odds ratios remained non-significant when the results were adjusted for confounding variables.

The analysis of pre-school care, which was restricted to those aged 5 or more at the time of diagnosis (children with diabetes) or qualifying (control chil- dren), is presented in Fig.1. The frequency of preschool care in the control groups varied widely between centres ranging from $17 \%$ in Luxemburg to $95 \%$ in Austria. The individual odds ratios associated with pre-school care were significantly less than one for three of the seven centres and the results approached significance for two of the remaining centres. The pooled odds ratio of $0.56(0.45,0.70)$ was highly statistically significant $(p<0.001)$. There was some evidence of heterogeneity mainly attributable to the results from the Luxemburg centre although this centre contributed little weight to the pooled odds ratio estimate. As shown at the foot of Fig. 1, adjustment for potential confounding variables made little difference to the reduction in the risk of diabetes associated with pre-school care.

Table 4. Odds ratios for parental recall of five common childhood infections before and after adjustment for confounding variables

\begin{tabular}{|c|c|c|c|c|}
\hline \multirow[t]{2}{*}{ Infection } & \multicolumn{2}{|c|}{$\begin{array}{l}\text { Mantel Haenszel analysis } \\
\text { stratified by centre }\end{array}$} & \multicolumn{2}{|c|}{$\begin{array}{l}\text { Logistic regression analysis adjusted } \\
\text { for confounders }{ }^{\mathrm{a}}\end{array}$} \\
\hline & Odds ratio $(95 \% \mathrm{CI})$ & $p$ & Odds ratio $(95 \% \mathrm{CI})$ & $p$ \\
\hline Morbilli/Measles & $1.16(0.91,1.47)$ & 0.23 & $1.00(0.73,1.38)$ & 0.98 \\
\hline Varicella/Chickenpox & $1.03(0.86,1.23)$ & 0.74 & $0.84(0.68,1.05)$ & 0.12 \\
\hline Pertussis/Whooping Cough & $1.01(0.70,1.45)$ & 0.96 & $0.99(0.63,1.55)$ & 0.96 \\
\hline Parotitis/Mumps & $1.20(0.93,1.55)$ & 0.16 & $1.14(0.84,1.54)$ & 0.40 \\
\hline
\end{tabular}

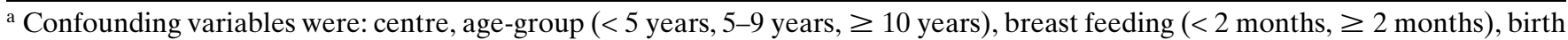
weight $(<2500 \mathrm{~g}, \geq 2500 \mathrm{~g})$, maternal age ( $\leq 25$ years, $>25$ years), jaundice at birth (yes, no), asthma before disease diagnosis (yes, no) and vitamin D supplementation (yes, no, unknown) 


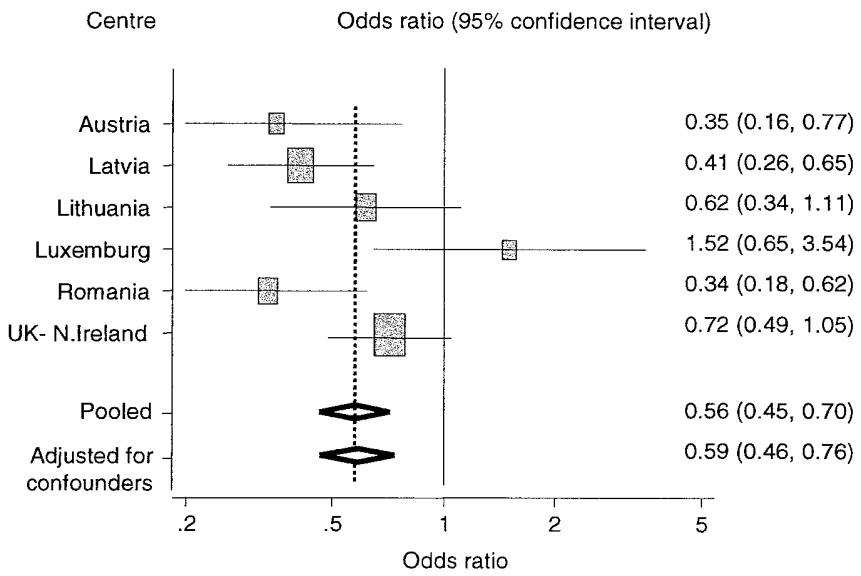

Fig. 1. Pooled odds ratio for pre-school day-care attendance with and without adjustment for confounding variables: the box size indicates the weight contributed by each centre to the Mantel-Haenszel pooled estimate

\section{Discussion}

This study shows that neonatal infectious diseases prospectively reported in hospital records are associated with a statistically significantly increased risk when adjusted for possible confounders. Because this significant finding was obtained in the context of multiple tests of hypothesis, some caution must, however, be exercised in its interpretation until corroborated by other studies. Nevertheless, the finding is consistent with previous studies showing a seasonality in the month of birth of children with diabetes [24] and simultaneous time and space clustering [25] suggesting that risk exposures operating around the time of birth and having an epidemic type of spread could be relevant. Furthermore, studies, from Sweden [8] and Finland [9] have shown an increase in comparison with control subjects' of coxsackie virus antibodies and antigens in maternal sera obtained during pregnancy from mothers of children who subsequently develop diabetes. The mechanism by which perinatal virus exposures could induce Type I diabetes has been debated since the observation that children with rubella embryopathy were at increased risk of diabetes. One possibility is that of a direct mimicry of autoantigens, this being supported by the observation that glutamic acid decarboxylase (GAD) carries sequence similarities not only with rubella virus capsid protein [26] but also to a sequence in the non-structural protein of coxsackie virus B [27]. Since the clinical onset of diabetes occurs several years after any early fetal or neonatal exposure, the early infection possibly only sensitises the organism through the GAD antigen and subsequent events could be necessary to activate the beta-cell destructive process. Such a mechanism could explain the widely reported occurrence of infectious disease just before the clinical onset of diabetes. On the other hand, fetal immunological events could have a specific effect on the developing immune system allowing other mechanisms to explain the association with early infections and autoimmunity. Due to lack of self/ non-self discrimination of the fetal immune system, fetal viral infections could lead to persisting infection either in the beta cell or in the vicinity of the beta cell which might cause a slowly progressing inflammation or autoimmune development [28]. The dynamics of the developing immune system also introduces the potential for differential effects of infectious disease exposures in different time periods. The hygiene hypothesis claims that normal "education" of the immune system depends on a certain load of infections during the first years of life to avoid the occurrence of atopic and autoimmune diseases later in life. Some have warned of the potential for vaccinations to increase the risk of diabetes [29], whereas others have shown in animal models that non-specific stimulation of natural supressor activity associated with BCG vaccine or complete Freund's adjuvant could be protective [30-32]. The possible role of vaccinations in insulin-dependent diabetes continues to be debated [33] but there is a lack of reliable data. Our study, one of the largest case-control studies yet conducted to address this issue, found no evidence to support vaccination modulating the risk of childhood diabetes. We also looked specifically to see if the timing of BCG vaccination had any influence but we found no support for the hypothesis that early BCG vaccination was protective.

In most previous studies a history of specific childhood infections has been reported as frequently in diabetic as in control children [2, 4, 5] although one study did report that chickenpox was less common in diabetic children [3]. The limitations of parental recall of childhood infections are, however, well-documented [34], so it is notable that a study which used general practitioner records as an information source found that infections in the first year of life were associated with a reduction in the risk of diabetes in childhood [35]. Another study that used information recalled by parents showed the same pattern although the finding did not attain statistical significance [4]. Our own data, also based on parental recall, showed no link with any specific infectious diseases. In the light of the reported inadequacy of parental recall of the frequency and timing of non-specific infectious episodes [34] we did not, however, attempt to record the total infectious disease experience in the early years of life. Instead we used the proxy measure of pre-school day-care attendance which is known to be associated with an increased burden of infectious disease $[36,37]$. We found that this measure had an inverse association with diabetes, a finding that is corroborated in a more detailed analysis of day-care arrangements in infancy in the study centre that we omitted from our analysis [21]. Contrary to our find- 
ings, pre-school day-care attendance has previously been associated with an increased risk of diabetes [2, 38 , although the findings in one study only just attained significance whereas in the other they were no longer significant after allowing for confounding variables. Since organised pre-school day-care has probably become more common in western European countries during the last few decades as mothers increasingly return to work after having their children, our finding of an inverse association with daycare attendance does not readily explain the recent widespread increase in the childhood diabetes incidence rate in European countries [39].

Other possible proxy measurements for infectious disease exposure in childhood have been studied. Three small-area analyses in the United Kingdom have reported lower rates of diabetes in children resident in areas of greatest material deprivation and of high population density [40-42], although an increased incidence in areas of material deprivation has also been reported [43]. Being the first child in the family could act as a marker for low exposure to infections, especially in the early years of life. Although generally birth order has not been found to be associated with diabetes $[3,44]$, there have been reports that diabetic children are more likely to be firstborn if attention is restricted to those with onset before the age of 5 years $[18,45]$. Family size has also been investigated and although there has been a report that diabetic children are more often from smaller families than control children [2] others have found no such association [46]. The current study provided no evidence to support an association with either birth order or family size.

The hypothesis that early exposure to infections can reduce the risk of diabetes has advocates [11, 47]. The epidemiological evidence is, however, still weak and the hypothesis must remain speculative even though there is clear evidence to support it from animal models. Prospective monitoring of infectious disease exposures among large cohorts of children may be necessary to provide a reliable answer to this question.

In conclusion, our large multicentre case-control study covering a wide range of maternal and neonatal infections as well as validated vaccination data supports previous evidence of early perinatal infections as being risk factors for childhood onset of Type I diabetes. A proxy measure of the total load of infections during the pre-school years, on the other hand, gives support for a protective effect perhaps through a specific effect on the developing immune system. Our study indicates that vaccinations do not exert any major modifying effect on the risk of Type I diabetes.

Acknowledgements. This study is part of the EURODIAB ACE study and has been supported in part by the European Community Concerted Action Programme (Grants BMH1-
CT92-0043 and BMH4-CT96-0577). Local grants were supplied from the Swedish Medical Research Council (project no. 07531), the British Diabetic Association, the Hungarian National Research Fund (project no. 019192) and CRP-Sauté, Luxemburg.

The EURODIAB Substudy 2 study group co-ordinators responsible for study design and preparation of the manuscript were: G. Dahlquist, Department of Clinical Science, Paediatrics, Umeå University, Umeå, Sweden; C. Patterson, Department of Epidemiology and Public Health, Queen's University Belfast, Northern Ireland, UK (also responsible for data processing and analysis); G. Soltész, Department of Paediatrics, University of Pecs, Hungary.

\section{The study centre leaders were:}

Austria: E. Schober and U. Schneider, Department of Paediatrics, University of Vienna, Vienna, Austria.

Bulgaria: S. Goranova, D. Stovanova and M. Ivanova, Children's Diabetic Centre, Sofia, Bulgaria

Lithuania: B. Urbonaite and A. Pundzuite, Department of Paediatrics, University of Kaunas, Kaunas, Lithuania.

Latvia: G. Brigis and I. Rubietza, Department of Public Health and Epidemiology, Medical Academy of Latvia, Riga, Latvia. Luxemburg: C. de Beaufort, Paediatric Clinic, Luxemburg

Romania: A.-M. Konnerth and K. Ionescu-Tirgoviste, Department of Nutrition and Metabolic Disease, University of Bucharest, Romania.

United Kingdom (Leeds): P. McKinney, Institute of Epidemiology and Health Services Research, University of Leeds, Leeds, UK.

United Kingdom (Northern Ireland): C. Patterson, R. Greenlees and D. Hadden, Department of Epidemiology and Public Health, Queen's University Belfast, Northern Ireland, UK.

\section{References}

1. Gundersen E (1927) Is diabetes of infectious origin? J Infect Dis 49: 197-202

2. Verge CF, Howard NJ, Irwig L, Simpson JM, Mackerras D, Silink M (1994) Environmental factors in childhood IDDM. A population-based, case-control study. Diabetes Care 17: 1381-1389

3. Glatthaar C, Whittall DE, Welborn TA et al. (1988) Diabetes in Western Australian children: descriptive epidemiology. Med J Aust 148: 117-123

4. Blom L, Nyström L, Dahlquist G (1991) The Swedish childhood diabetes study. Vaccinations and infections as risk determinants for diabetes in childhood. Diabetologia 34: $176-181$

5. Soltész G, Jeges S, Dahlquist G (1994) Non-genetic risk determinants for type 1 (insulin-dependent) diabetes mellitus in childhood. Acta Paediatr 83: 730-735

6. Levy-Marchal C, Patterson C, Green A (1995) Variation by age-group and seasonality at diagnosis of childhood IDDM in Europe. Diabetologia 38: 823-830

7. Menser MA, Forrest JM, Bransby RD (1978) Rubella infection and diabetes mellitus. Lancet 8055: 57-60

8. Dahlquist G, Frisk G, Ivarsson SA, Svanberg L, Forsgren M, Diderholm H (1995) Indications that maternal coxsackie B virus infection during pregnancy is a risk factor for childhood-onset IDDM. Diabetologia 38: 1371-1373

9. Hyöty H, Hiltunen M, Knip M et al. (1995) A prospective study of the role of coxsackie B and other enterovirus infections in the pathogenesis of IDDM. Diabetes 44: 652-657 
10. Dahlquist GG, Boman J, Juto P (1999) Enteroviral RNA and IgM antibodies in early pregnancy and risk for childhood onset IDDM in offspring. Diabetes Care 22: 364-365

11. Rook GAW, Stanford JL (1998) Give us this day our daily germs. Immunol Today 19: 113-116

12. Todd JA (1991) A protective role of the environment in the development of Type 1 diabetes. Diabet Med 8: 906-910

13. Dahlquist G, Gothefors L (1995) The cumulative incidence of childhood diabetes mellitus in Sweden unaffected by BCG vaccination (letter). Diabetologia 38: 873-874

14. Heijbel H, Chen RT, Dahlquist G (1997) Cumulative incidence of childhood-onset IDDM is unaffected by pertussis immunization. Diabetes Care 20: 173-175

15. Hyöty H, Hiltunen M, Reunanen A et al. (1993) Decline of mumps antibodies in Type 1 (insulin-dependent) diabetic children and a plateau in the rising incidence of Type 1 diabetes after introduction of the mumps-measles-rubella vaccine in Finland. Diabetologia 36: 1303-1308

16. Karvonen M, Cepaitis Z, Tuomilehto J (1999) Association between Type 1 diabetes and Haemophilus influenzae type b vaccination: birth cohort study. BMJ 318: 11691172

17. Parent ME, Siemiatycki J, Menzies R, Fritschi L, Colle E (1997) Bacille Calmette-Guerin vaccination and incidence of IDDM in Montreal, Canada. Diabetes Care 20: 767772

18. Wadsworth EJK, Shield JPH, Hunt LP, Baum JD (1997) A case-control study of environmental factors associated with diabetes in the under 5 s. Diabet Med 14: 390-396

19. Green A, Gale EAM, Patterson CC (1992) Incidence of childhood-onset insulin-dependent diabetes mellitus: the EURODIAB ACE study. Lancet 339: 905-909

20. The EURODIAB Substudy 2 Study Group (1999) Vitamin D supplement in early childhood and risk for Type 1 (insulin-dependent) diabetes mellitus. Diabetologia 42: 51-54

21. McKinney PA, Okasha M, Parslow RC et al. (1999) Early social mixing at day care protects against childhood diabetes. Diabetologia 42[Suppl 1]:A74 (Abstract)

22. Breslow NE, Day NE (1980) Statistical methods in cancer research. Vol 1. The analysis of case-control studies. International Agency for Research on Cancer, Lyons

23. The EURODIAB Substudy 2 Study Group (1999) Perinatal risk factors for childhood IDDM in Europe. Diabetes Care 22: 1698-1702

24. Rothwell PM, Staines A, Smail P, Wadsworth E, McKinney P (1996) Seasonality of birth of patients with childhood diabetes in Britain. BMJ 312: 1456-1457

25. Dahlquist GG, Källén BAJ (1996) Time-space clustering of date at birth in childhood-onset diabetes. Diabetes Care 19: 328-332

26. Karounos DG, Wolinsky JS, Thomas JW (1993) Monoclonal antibodies to rubella virus capsid protein recognizes a beta-cell antigen. J Immunol 150: 3080-3085

27. Kaufman DL, Erlander MG, Clare-Salzler M, Atkinson MA, MacLaren NK, Tobin AJ (1992) Autoimmunity to two forms of glutamate decarboxylase in insulin-dependent diabetes mellitus. J Clin Invest 89: 283-292

28. Greenbaum CJ, Brooks-Worrel BM, Palmer JP, Lernmark $\AA$ (1994) Autoimmunity and prediction of insulin-dependent diabetes mellitus. In: Marshall SM, Home PH (eds) The diabetes annual VIII. Elsevier, Amsterdam
29. Classen DC, Classen JB (1997) The timing of pediatric immunization and the risk of insulin-dependent diabetes mellitus. Infect Dis Clin Pract 6: 449-454

30. Harada M, Kishimoto Y, Makino S (1990) Prevention of overt diabetes and insulitis in NOD mice by a single BCG vaccination. Diabetes Res Clin Pract 8: 85-89

31. Sadelain MWJ, Qin H-Y, Lauzon J, Singh B (1990) Prevention of Type I diabetes in NOD mice by adjuvant immunotherapy. Diabetes 39: 583-589

32. Shehadeh N, Calcinaro F, Bradley BJ, Bruchlim I, Vardi P, Lafferty KJ (1994) Effect of adjuvant therapy on development of diabetes in mouse and man. Lancet 343: 706-707

33. Jefferson T (1998) Vaccination and its adverse effects: real or perceived. BMJ 317: 159-160

34. McKinney PA, Alexander FE, Nicholson C, Cartwright RA, Carette J (1991) Mothers' reports of childhood vaccinations and infections and their concordance with general practitioner records. J Public Health Med 13: 13-22

35. Gibbon C, Smith T, Egger P, Betts P, Phillips D (1997) Early infection and subsequent insulin-dependent diabetes. Arch Dis Child 77: 384-385

36. Osterholm MT (1994) Infectious disease in child day care: an overview. Pediatrics 94: 987-990

37. The Child Day Care Infectious Disease Study Group (1984) Public health considerations of infectious diseases in child day care centres. J Pediatr 105: 683-701

38. Siemiatycki J, Colle E, Campbell S, Dewar RAD, Belmonte MM (1989) Case-control study of IDDM. Diabetes Care 12: 209-216

39. The EURODIAB TIGER Study Group (1998) Recent trends in the incidence of Type 1 diabetes in European children. Diabetologia 41[Suppl 1]:A21 (Abstract)

40. Patterson CC, Waugh NR (1992) Urban/rural and deprivational differences in incidence and clustering of childhood diabetes in Scotland. Int J Epidemiol 21: 108-117

41. Patterson CC, Carson DJ, Hadden DR (1996) Epidemiology of childhood IDDM in Northern Ireland 1989-1994: Low incidence in areas with highest population density and most household crowding. Diabetologia 39: 1063-1069

42. Staines A, Bodansky HJ, McKinney PA et al. (1997) Small area variation in the incidence of childhood insulin-dependent diabetes mellitus in Yorkshire, UK: links with overcrowding and population density. Int $\mathrm{J}$ Epidemiol 26: 1307-1313

43. Crow YJ, Alberti KGMM, Parkin JM (1991) Insulin-dependent diabetes in childhood and material deprivation in northern England, 1977-86. BMJ 303: 158-160

44. Bock T, Pedersen CR, Vølund A, Pallesen CS, Buschard K (1994) Perinatal determinants among children who later develop IDDM. Diabetes Care 17: 1154-1157

45. Patterson CC, Carson DJ, Hadden DR, Waugh NR, Cole SK (1994) A case-control investigation of perinatal risk factors for childhood IDDM in Northern Ireland and Scotland. Diabetes Care 17: 376-381

46. Blom L, Dahlquist G, Nyström L, Sandström A, Wall S (1989) The Swedish childhood diabetes study - social and perinatal determinants for diabetes in childhood. Diabetologia 32: 7-13

47. Kolb H, Elliott RB (1994) Increasing incidence of IDDM a consequence of improved hygiene? (letter), Diabetologia 37: 729 